\title{
Relación entre trastornos neurocognitivos, Diabetes Mellitus Tipo 2 y otros factores en adultos mayores del Centro Médico Naval del Perú, entre los años 2010 a 2015
}

Relationship between Type 2 Diabetes Mellitus and cognitive impairment in Peru's Navy Medical Center's older adult patients between 2010 to 2015.

Daniela Zapata-Tragodara ${ }^{1}$, Helaman Roque ${ }^{1}$, Fernando M. Runzer-Colmenares ${ }^{1,2}$, José F. Parodi ${ }^{2}$

\section{RESUMEN}

Diabetes Mellitus (DM) se caracteriza fisiopatológicamente por alteraciones metabólicas tales como hiperglucemia crónica debida a un defecto en la secreción de insulina, en la acción de ésta o a una combinación de ambos factores. Los trastornos neurocognitivos o trastornos mentales orgánicos presentan tres categorías: delirium, trastorno neurocognitivo menor (para referirse a deterioro cognitivo leve) y trastorno neurocognitivo mayor, este último mejor conocido como demencia. Varios estudios epidemiológicos sobre trastornos neurocognitivos y DM han mostrado algunas características comunes a ambas patologías. Objetivo: Determinar la relación entre DM y trastornos neurocognitivos en la población adulta mayor. Material y Métodos: Estudio retrospectivo con análisis secundario de bases de datos procedentes de investigaciones realizadas entre los años 2010 y 2015 en una población de 1896 adultos mayores, atendidos en el Servicio de Geriatría del Centro Médico Naval, el principal establecimiento hospitalario de la Marina de Guerra del Perú. Resultados: Se encontró una frecuencia mayor de pacientes del sexo masculino (58,4\%), el grupo etario más frecuente de 71-80 años $(46,4 \%)$ y una frecuencia de DM del 16,86\%. En el análisis bivariado, un 30,67\% de participantes con trastorno neurocognitivo tenían antecedente de Diabetes, con diferencias estadísticamente significativas. Una similar asociación estadística fue evidente en cuanto a variables como edad, sexo, educación, apoyo social, polifarmacia, sedentarismo y promedio de hemoglobina glicosilada (7.03). Conclusiones: Se documentó una relación entre Diabetes Mellitus y deterioro cognitivo. Sexo masculino parece constituír un factor protector de trastornos neurocognitivos. La hiperglucemia estaría relacionada con estos últimos y la hemoglobina glicosilada puede ser un test determinante en la valoración del adulto mayor diabético con problemas cognitivos.

PALABRAS CLAVE: Demencia, diabetes mellitus, geriatría, envejecimiento.

\section{SUMMARY}

Diabetes Mellitus (DM), a metabolic disorder characterized by chronic hyperglycemia, can be due to a defect in insulin secretion, a defect in its action, or both. Neurocognitive disorders or "organic mental disorders" present three categories: delirium, minor (or mild) impairment, and major neurocognitive disorder or dementia. These are two very prevalent conditions; several epidemiological studies have shown some characteristics common to both of

Universidad Científica del Sur. Lima, Perú.

2 Centro de Investigación del Envejecimiento, Facultad de Medicina Humana, Universidad de San Martín de Porres. Lima, Perú. 
them. Objective: To determine the relationship between Type $2 \mathrm{DM}$ and neurocognitive disorders in an older adult population. Material and Methods: Analytical, retrospective, and secondary database study of investigations carried out between 2010 and 2015 in a population of 1,896 older adults, attended in the Geriatric service of the Naval Medical Center, the main hospital of the Peruvian Navy. Results: A higher frequency of male patients (58.4\%) was found, the most frequent age group (46.4\%) was 71-80 years, and the frequency of DM was $16.86 \%$. In the bivariate analysis, $30.67 \%$ of participants with neurocognitive disorders had a history of DM, with statistically significant differences; in addition, variables such as age, sex, education, social support, polypharmacy, sedentary lifestyle and 7.03 average of glycosylated hemoglobin were also statistically significant. Conclusions: A relationship was found between DM and neurocognitive disorders. Being male seems to be a protective factor against cognitive decline. Hyperglycemia causes cognitive impairment, with glycosylated hemoglobin being a determinant test in the assessment of older diabetics with cognitive problems.

KEYWORDS: Dementia, diabetes mellitus, geriatrics, aging.

\section{INTRODUCCIÓN}

La diabetes mellitus es una enfermedad crónica muy frecuente, considerada también un problema de salud pública. Incluye presentaciones clásicas con glucemia en ayunas igual o mayor de $126 \mathrm{mg} / \mathrm{dL}$ y glucemia casual, igual o mayor a $200 \mathrm{mg} / \mathrm{dL}$, definiendo como ayuno a la falta de ingesta calórica de, al menos, ocho horas. Las complicaciones de la diabetes son múltiples, como enfermedad cardiovascular, cerebrovascular, microvascular, neuropatías, y en algunos contextos, Enfermedad de Alzheimer, problema cognitivo más frecuente $(1,2)$. Se han propuesto conceptos de alteración cognitiva asociada a la diabetes mellitus previa a la demencia, como la disfunción cognitiva asociada a la diabetes, durante la cual los pacientes presentan quejas subjetivas de memoria, con una alteración sutil en las pruebas neuropsicológicas; y deterioro cognitivo leve (DCL). Los afectados por DCL tienen problemas de memoria, pero pueden desarrollar las actividades cotidianas sin problemas $(3,4,5)$.

La diabetes mellitus según cifras de la Organización Mundial de la Salud (OMS) representa a la actualidad una pandemia, con cifras de 422 millones de personas afectadas y una prevalencia $8,5 \%$, a su vez en Latinoamérica y el Perú según los últimos reportes de la Asociación Latinoamericana de la Diabetes (ALAD) se presentan frecuencias de 9,2\% y 5.6\%, respectivamente, en personas entre los 20 a 79 años de edad (4-6), resaltando la importancia de explorar sus potenciales relaciones con otras enfermedades a nivel nacional (6).

A nivel mundial, se describe en neuroimágenes de pacientes diabéticos la disminución del volumen cerebral que se da por atrofia cortical y subcortical $\mathrm{y}$, como consecuencia, aumento del tamaño de los ventrículos cerebrales. Análisis regionales del cerebro demostraron que el territorio más comprometido era el lóbulo temporal medial, y en particular el hipocampo. También se describe compromiso de la corteza prefrontal, principalmente en el cíngulo anterior y la región orbito-frontal (7).

$\mathrm{Al}$ respecto, el TNC, se define como un trastorno mental que afecta a las funciones neurocognitivas, principalmente la memoria, la percepción y la resolución de problemas; clasificados como: delirium, trastorno neurocognitivo mayor (conocido como demencia) y trastorno neurocognitivo menor (conocido como deterioro cognitivo leve)(8-10). Los TNC más directos incluyen: la amnesia, demencia(trastorno neurocognitivo mayor) y delirium (11-14).

Existe evidencia de que la población adulta mayor en nuestro país y a nivel mundial aumenta de forma acelerada, en gran medida debido a la mejoría de la atención médica global y otros factores. Actualmente la prevalencia de demencia antes de la edad de 65 años es de entre 2 al $10 \%$, pero por cada cinco años que transcurren se duplica $(14,15)$. Los factores de riesgo de TNC están relacionados con aspectos etiológicos o etiopatogénicos de la enfermedad, se incluyen los factores demográficos como la edad, duplicándose cada cinco años hasta que el adulto mayor cumple los 85 años; los factores genéticos como el gen de la apolipoproteína E para los TNC; factores cardiovasculares donde encontramos a la diabetes mellitus, altos niveles de glucemia y deficiencia o resistencia a la insulina se han asociado a un incremento en el riesgo $(16,17)$. El aumento de la presión arterial incrementa el riego de deterioro cognitivo. Los factores sensoriales pueden impactar en la relación de la persona con su entorno. El déficit 
auditivo se ha relacionado con la fragilidad física y cognitiva. Los estilos de vida han mostrado que hay asociación con la función cognitiva, el consumo de tabaco y el consumo de alcohol (17-20).

La diabetes mellitus y los TNC son dos condiciones muy prevalentes actualmente, varios estudios epidemiológicos acerca del daño cognitivo y la diabetes mellitus han mostrado algunas características comunes entre ambas patologías, así se ha propuesto el término diabetes mellitus tipo 3 para dar una visión integradora de los posibles mecanismos patológicos entre la diabetes mellitus y la Enfermedad de Alzheimer que es el TNC más frecuente a nivel mundial. Se han descrito diversas hipótesis que intentan explicar la relación entre las dos patologías ya antes mencionadas (21-24). Si bien la relación epidemiológica entre diabetes mellitus y TNC se encuentra establecida, aún no se puede reconocer la fisiopatología, se discute en torno a dos mecanismos, el origen vascular y el origen no vascular ligado al metabolismo del b-amiloide $(\mathrm{AB})$ y la proteína Tau. En el origen vascular se hace alusión a la diabetes y la proteína al metabolismo del $\mathrm{AB}$ y la proteína tau como factor de riesgo vascular, esto subyace como causa, en la demencia vascular pero además disminuye el umbral de acumulación de B-amiloide, aumentando la frecuencia de enfermedad de sustancia blanca o leucoaraiosis(25).

La patogenia no vascular es explicada, entre otros factores, a partir de la hiperinsulinemia, puesto que en el encéfalo existen receptores de insulina, con más frecuencia en el hipocampo y corteza, las cuales son regiones que se afectan en la Enfermedad de Alzheimer precozmente. La insulina atraviesa la barrera hematoencefálica y su infusión periférica se ha asociado el aumento de marcadores de riesgo para la Enfermedad de Alzheimer (26). The Honolulu Asia Aging Study concluyó que existe una relación mucho más estrecha entre la diabetes mellitus y la Enfermedad de Alzheimer si es que el paciente es portador del alelo APOE-E4, puesto que los portadores tienen menor capacidad de reparación neuronal y actividad antioxidante $(27,28)$.

Se han postulado otras hipótesis que sostienen la evidencia existente, entre estas las de mayor relevancia son la hiperglicemia, la hipoglicemia, la resistencia a la insulina y el depósito de material amiloide (14,23-28). Además, se cree que la hiperglicemia podría conducir a alteraciones metabólicas como la activación de la vía de los polioles o la formación de productos de glicación avanzada, los cuales podrían estar relacionados con un declive de la función cognitiva. También se postulan como factores de riesgo niveles elevados de hemoglobina glicosilada (HbA1c) y un pobre control glicémico. Hasta la fecha no se ha establecido un test óptimo para evaluar TNC en pacientes con diabetes, sin embargo, pueden usarse el Montreal Cognitive Assessment (MoCA) test y el Minimental State Examination (26-28).

Existen relaciones, vías y eventos fisiopatológicos comunes entre la condición de fragilidad, diabetes tipo 2 y la evolución de TNC que se han descrito en la literatura. Mencionamos esto para recordar que existen metas terapéuticas que se ajustan en personas mayores por estas variables e intervenciones comunes como el ejercicio multidominio que surgen como las opciones más convenientes de cara a la atención centrada en la persona más que en la enfermedad $(28,29)$.

En los últimos años se ha prestado especial atención a la asociación entre diabetes y deterioro cognitivo lo cual puede verse reflejado en varios estudios realizados hasta la fecha $(14-17,21,29)$, siendo el más importante el de Xiaojun Zhang et al, donde encontraron un $\mathrm{OR}=$ 1,45 (IC: 95\% [1,25, 1,69] para estudios transversales analíticos (21), lo que refuerza la asociación entre diabetes mellitus tipo 2 y trastornos neurocognitivos; sin embargo, algunos estudios presentan discrepancias como los estudios de Roberts et al., Saito et al., y Winkler, cuyos resultados no fueron estadísticamente significativos en relación a asociación entre diabetes y TNC (22-24).

En nuestra revisión bibliográfica se encontraron escasos estudios a nivel de Latinoamérica o nacionales que exploren la asociación entre la diabetes mellitus tipo 2 con el trastorno neurocognitivo en población adulta mayor, y tomando en cuenta las características propias de nuestra población, el objetivo principal del presente artículo es determinar la asociación entre la diabetes mellitus tipo 2 y los trastornos neurocognitivos en adultos mayores. Si bien la diabetes mellitus es un factor de riesgo para trastorno neurocognitivo no todos los pacientes con esta enfermedad estarán predispuestos a padecerlos, debemos tener en cuenta que existen otros factores como el tiempo de enfermedad, el control de la diabetes mellitus que pueden actuar como factores de riesgo para el desarrollo de dichos trastornos. Además, no existen suficientes estudios a nivel de Latinoamérica donde se compruebe el impacto de la hemoglobina glicosilada como un factor para saber si la diabetes mellitus mal controlada está considerada o no un factor de riesgo para el desarrollo de trastornos cognitivos. 


\section{MATERIAL Y METODOS}

El presente estudio es de tipo analítico, retrospectivo. Análisis secundario de bases de datos.

Los datos para el presente análisis provienen de un estudio realizado entre los años 2010 y 2015 en una población de 1896 adultos mayores, atendidos en el servicio de Geriatría del Centro Médico Naval, que es un hospital de la Marina de Guerra del Perú, especializado. El objetivo de dicho estudio fue determinar factores asociados a fragilidad. Los criterios de inclusión fueron: edad 60 años o más, vivir en Lima o Callao. Se excluyeron a pacientes en atención domiciliaria, hospitalizados, con diagnóstico de cáncer y VIH-SIDA (27).

No se calculó tamaño muestral para el presente estudio, pues utilizamos todos los datos de los participantes de dicho estudio. Contamos con un tamaño muestral de 1868 luego de aplicar los criterios de elegibilidad. Utilizando el software Open Epi versión 3.0 asumiendo una frecuencia de problemas cognitivos en pacientes diabéticos del 37,5\% (28), y asumiendo una frecuencia de problemas cognitivos en población adulta mayor general del $22,73 \%$ (29), con un intervalo de confianza del $95 \%$, la potencia estadística asciende al 100\%.

Criterios de inclusión: Todos los datos de participantes que se encontraron en la base de datos.

Criterios de exclusión: Datos faltantes de variables principales del estudio, cabe resaltar que se eliminaron datos de 28 participantes del estudio por no tener la variable diabetes mellitus.

\section{Variables :}

Dependiente:

- Trastorno neurocognitivo: En el estudio original se utilizó la escala de Montreal Cognitive Assesment versión en Español, para evaluar problemas cognitivos en la línea de base del estudio, consideramos un puntaje mayor o igual a 26 puntos como normal, considerando un punto adicional si el paciente tenía educación de 11 años o menos (30).

\section{Independiente:}

- Diabetes Mellitus: Considerado como antecedente de diabetes reportado en la Historia Clínica de los pacientes del estudio original.
Tabla 1. Análisis descriptivo de las variables de estudio. $(\mathrm{n}=1868)$

\begin{tabular}{|c|c|c|}
\hline Edad & n & $\%$ \\
\hline 60-70 años & 283 & 15,15 \\
\hline 71-80 años & 866 & 46,36 \\
\hline$>80$ años & 719 & 38,49 \\
\hline \multicolumn{3}{|l|}{ Sexo } \\
\hline Mujer & 775 & 41,6 \\
\hline Hombre & 1,088 & 58,4 \\
\hline \multicolumn{3}{|l|}{ Educación } \\
\hline Técnica Superior & 538 & 30,62 \\
\hline Colegio incompleto/completo & 1219 & 69,38 \\
\hline \multicolumn{3}{|l|}{ Estado civil } \\
\hline Soltero & 49 & 2,87 \\
\hline Casado & 1253 & 73,4 \\
\hline Viudo & 340 & 19,92 \\
\hline Separado/Divorciado & 65 & 3,81 \\
\hline \multicolumn{3}{|l|}{ Comorbilidades } \\
\hline Ninguna & 53 & 3,36 \\
\hline 1 & 179 & 11,35 \\
\hline 2 o más & 1345 & 85,29 \\
\hline \multicolumn{3}{|l|}{ Dependencia funcional } \\
\hline No & 620 & 33,86 \\
\hline $\mathrm{Si}$ & 1211 & 66,14 \\
\hline \multicolumn{3}{|l|}{ Soporte Social } \\
\hline Adecuado & 416 & 22,27 \\
\hline Inadecuado & 1452 & 77,73 \\
\hline \multicolumn{3}{|l|}{ Polifarmacia } \\
\hline No & 1160 & 65,32 \\
\hline $\mathrm{Si}$ & 616 & 34,68 \\
\hline \multicolumn{3}{|l|}{ Sedentarismo } \\
\hline No & 796 & 49,78 \\
\hline $\mathrm{Si}$ & 803 & 50,22 \\
\hline \multicolumn{3}{|l|}{ Diabetes Mellitus } \\
\hline No & 1553 & 83,14 \\
\hline $\mathrm{Si}$ & 315 & 16,86 \\
\hline
\end{tabular}

\section{Co-variables:}

Sociodemográficas:

- Edad: donde se divide a los pacientes en: 60-70 años, 71-80 años, mayores de 80 años.

- Sexo: femenino, masculino.

- Educación: 11 años o menos, más de 11 años.

- Estado civil: soltero(a), casado(a), viudo(a), divorciado(a)/separado(a). 
- Comorbilidades medicas: donde se categorizó en: ninguna, una, dos o más. Las comorbilidades que se consideraron para este estudio fueron: antecedente de enfermedad renal crónica, hipertensión arterial, Enfermedad Pulmonar Obstructiva Crónica (EPOC), hipotiroidismo, Insuficiencia Cardiaca Congestiva (ICC), depresión, Desorden Cerebro Vascular (DCV), además auto reporte de consumo de tabaco, alcohol y obesidad definido como IMC mayor o igual a 30.

- Índice de Barthel: Para evaluar actividades de la vida diaria, se consideró un puntaje menor de 100 puntos como presencia de dependencia (31).

- Soporte social: Se utilizó una pregunta del cuestionario de Edmonton para evaluar el soporte social en los pacientes (32).

- Polifarmacia: Consumo de 5 fármacos o más (33).

- Sedentarismo: Se definió según la escala Physical Activity Scale for the Elderly (PASE), en esta escala se mide actividad física en diferentes aspectos y utiliza un punto de corte en mujeres menor de 64 y en varones menor de 52 (34).

- Valores de Hemoglobina Glicosilada: Expresados en porcentaje, muestras tomadas al inicio del estudio; tanto en pacientes diabéticos como en pacientes no diabéticos.

\section{Procedimiento}

Se consiguió el permiso del uso de la base de datos por parte del Centro de Investigación del Envejecimiento de la Universidad de San Martin de Porres, posteriormente se hizo el análisis estadístico donde en un inicio se describieron las variables según su porcentaje, frecuencia, promedio y desviación estándar para las variables numéricas. A continuación, se hizo un análisis bivariado utilizando Chi cuadrado para variables categóricas y $\mathrm{T}$ de Student para variables numéricas. Finalmente se incluyeron las variables significativas en una regresión de Poisson para determinar razones de prevalencia e intervalos de confianza al $95 \%$ en modelos crudos y ajustados, incluyendo variables confusoras con criterio estadístico. Se utilizó Stata versión 15.0 para el análisis estadístico (tabla 1).

\section{RESULTADOS}

En el presente estudio encontramos una frecuencia mayor de pacientes del sexo masculino con $58,4 \%$, el grupo etario más frecuente fue el de 71-80 años que representó el $46.4 \%$ de participantes del estudio, una frecuencia de diabetes mellitus del $16,86 \%$.
Además, en el análisis bivariado encontramos que un $30,67 \%$ de participantes con trastorno neurocognitivo tenían antecedente de diabetes mellitus, con diferencias estadísticamente significativas; adicional a ello variables como la edad, el sexo, la educación, el soporte social, la polifarmacia, el sedentarismo y el promedio de hemoglobina glicosilada salieron asociados estadísticamente en el análisis bivariado.

Se construyeron tres modelos de regresión para determinar la asociación entre diabetes mellitus y trastornos neurocognitivos, en un modelo crudo evidenciamos que en pacientes con diabetes mellitus se incrementa la probabilidad de trastorno neurocognitivo en 2.2 veces con un intervalo de confianza que va entre 2,06 y 2,31 (tabla 2). En el modelo número 2 ajustado por covariables significativas en el análisis bivariado encontramos como factores asociados al sexo masculino como factor protector y a la educación escolar completa o incompleta como factor de riesgo en comparación un grado de instrucción técnico/superior.

En el modelo final que fue ajustado según valores de hemoglobina glicosilada, determinamos que la diabetes mellitus duplica las probabilidades de tener trastornos neurocognitivos, independientemente de los valores de hemoglobina glicosilada y adicionalmente encontramos que a mayor promedio de hemoglobina glicosilada la probabilidad de tener trastorno neurocognitivo fue mayor, independientemente de si los participantes eran diabéticos o no.

\section{DISCUSIÓN}

El objetivo del presente estudio fue determinar la asociación entre diabetes mellitus y trastorno neurocognitivos; además de explorar otras covariables como hemoglobina glicosilada $(34,35)$.

Encontramos que si hubo una asociación entre la diabetes mellitus y TNC, además que los valores de hemoglobina glicosilada también se asociaron a mayor frecuencia de trastorno neurocognitivo. Un estudio en el Ecuador describe una realidad parecida, nos describe prevalencia de trastorno neurocognitivo en pacientes con diabetes mellitus, los cuales fueron evaluados con la escala MoCA. En Argentina existe un estudio en el que MoCA evalúa la relación entre la demencia y diabetes mellitus, demostrando que si es un factor de riesgo (38).

Ser del sexo masculino se consideró un factor 
Tabla 2. Regresión de Poisson para determinar asociación entre Diabetes Mellitus y Trastornos Neurocognitivos(n=1868)

\begin{tabular}{|c|c|c|c|}
\hline Variables & MODELO 1 & MODELO 2 & MODELO 3 \\
\hline \multicolumn{4}{|l|}{ Edad } \\
\hline 60-70 años & Referencia & Referencia & \\
\hline $71-80$ años & $1,12(0,97-2,28)$ & $0,99(0,87-1,13)$ & \\
\hline$>80$ años & $1,20(1,05-1,38)$ & $1,05(0,92-1,20)$ & \\
\hline \multicolumn{4}{|l|}{ Sexo } \\
\hline Mujer & Referencia & Referencia & \\
\hline Hombre & $0,92(0,84-0,99)$ & $0,91(0,83-0,99)$ & \\
\hline \multicolumn{4}{|l|}{ Educación } \\
\hline Técnica Superior & Referencia & Referencia & \\
\hline Colegio incompleto/completo & $0,85(0,78-0,94)$ & $1,11(1,01-1,22)$ & \\
\hline \multicolumn{4}{|l|}{ Soporte Social } \\
\hline Inadecuado & Referencia & Referencia & \\
\hline Adecuado & $0,89(0,81-0,98)$ & $1,09(0,96-1,24)$ & \\
\hline \multicolumn{4}{|l|}{ Polifarmacia } \\
\hline No & Referencia & Referencia & \\
\hline $\mathrm{Si}$ & $1,87(1,72-2,03)$ & $2,01(1,83-2,21)$ & \\
\hline \multicolumn{4}{|l|}{ Sedentarismo } \\
\hline No & Referencia & Referencia & \\
\hline $\mathrm{Si}$ & $1,54(1,39-1,69)$ & $1,28(1,16-1,41)$ & \\
\hline \multicolumn{4}{|l|}{ Diabetes Mellitus } \\
\hline No & Referencia & Referencia & Referencia \\
\hline $\mathrm{Si}$ & $2,18(2,06-2,31)$ & $2,45(2,26-2,64)$ & $2,04(1,88-2,20)$ \\
\hline \multicolumn{4}{|l|}{ Hemoglobina glicosilada } \\
\hline Valores de $\mathrm{Hb}$ glicosilada (\%) & $1,33(1,29-1,37)$ & & $1,05(1,02-1,09)$ \\
\hline
\end{tabular}

protector. Posiblemente el principal factor sea de salud cardiovascular, hormonal o quizás que en la generación actual de adultos mayores varones haya tenido una exposición menor al analfabetismo y pobre educación en comparación a las mujeres. $(30,36)$, cuyos resultados coinciden con lo encontrado en nuestros resultados, en el mismo artículo se describe como factor de riesgo la educación pobre; los pacientes con educación secundaria completa o incompleta, tenían una frecuencia $11 \%$ mayor de tener trastornos neurocognitivos en comparación a los pacientes que tenían educación superior, lo cual concuerda con la literatura consultada $(30,35-39)$.

Por otro lado, la polifarmacia se vuelve un factor de riesgo puesto que los adultos mayores sufren cambios fisiológicos asociados al envejecimiento, según un estudio de Colombia; la polifarmacia actúa como predictor para desarrollo de trastorno neurocognitivo en pacientes adultos mayores, y los factores que se asocian más a la polifarmacia son las enfermedades crónicas, el grado de dependencia para las actividades básicas de la vida diaria $(40,41)$. Un alto porcentaje de los pacientes adultos mayores que acuden a la consulta médica consume al menos un medicamento de forma crónica (42). En el estudio SABE (Salud, Bienestar y Envejecimiento) realizado en Brasil en 1115 adultos de 65 años o más, la polifarmacia fue definida como el uso de cinco o más medicamentos, encontrando una prevalencia del $36 \%$ donde el sexo femenino, ser mayor de 75 años, ingresos económicos más altos, ser activo desde el punto de vista laboral, peor auto reporte de salud, diagnóstico de hipertensión, diabetes, enfermedades reumáticas 
Tabla 3. Análisis bivariado en base a presencia de trastornos neurocognitivos ( $\mathrm{n}=1868)$

\begin{tabular}{|c|c|c|c|}
\hline Variables & $\begin{array}{c}\text { Sin Trastornos } \\
\text { Neurocognitivos } n(\%)\end{array}$ & $\begin{array}{c}\text { Con Trastornos } \\
\text { Neurocognitivos } n(\%)\end{array}$ & Valor de $P$ \\
\hline Edad & & & 0,021 \\
\hline 60-70 años & $149(17,19)$ & $134(13,39)$ & \\
\hline 71-80 años & $408(47,06)$ & $458(45,75)$ & \\
\hline$>80$ años & $310(35,76)$ & $409(40,86)$ & \\
\hline Sexo & & & 0,042 \\
\hline Mujer & $337(39,10)$ & $438(43,76)$ & \\
\hline Hombre & $525(60,90)$ & $563(56,24)$ & \\
\hline Educación & & & 0,001 \\
\hline Técnica Superior & $223(26,80)$ & $315(34,05)$ & \\
\hline Colegio incompleto/completo & $609(73,20)$ & $610(65.95)$ & \\
\hline Estado civil & & & 0,085 \\
\hline Soltero & $21(2,56)$ & $28(3,16)$ & \\
\hline Casado & $607(73,93)$ & $646(72,91)$ & \\
\hline Viudo & $153(18,64)$ & $187(21.11)$ & \\
\hline Separado/Divorciado & $40(4,87)$ & $25(2.82)$ & \\
\hline Comorbilidades & & & 0,782 \\
\hline Ninguna & $27(3,54)$ & $26(3,19)$ & \\
\hline 1 & $90(11,81)$ & $89(10,92)$ & \\
\hline 2 o más & $645(84,65)$ & $700(85,89)$ & \\
\hline Dependencia Funcional & & & 0,757 \\
\hline No & $294(34,23)$ & $326(33,54)$ & \\
\hline $\mathrm{Si}$ & $565(65,77)$ & $646(66,46)$ & \\
\hline Soporte Social & & & 0,025 \\
\hline Adecuado & $173(19,95)$ & $243(24,28)$ & \\
\hline Inadecuado & $694(80,05)$ & $758(75,72)$ & \\
\hline Polifarmacia & & & 0,001 \\
\hline No & $688(82,40)$ & $472(50,16)$ & \\
\hline $\mathrm{Si}$ & $147(17,60)$ & $469(49,84)$ & \\
\hline Sedentarismo & & & 0,001 \\
\hline No & $468(61,34)$ & $328(39,23)$ & \\
\hline $\mathrm{Si}$ & $295(38,66)$ & $508(60,77)$ & \\
\hline Diabetes Mellitus & & & 0,001 \\
\hline No & $859(99,08)$ & $694(69,33)$ & \\
\hline $\mathrm{Si}$ & $8(0,92)$ & $307(30,67)$ & \\
\hline $\begin{array}{l}\text { Hemoglobina glicosilada } \\
\text { Media(desviación estándar)(\%) }\end{array}$ & $6,6(0,48)$ & $7,03(0,91)$ & 0,001 \\
\hline
\end{tabular}


y problemas cardiacos, fueron asociados con una mayor prevalencia de polifarmacia. Por el contrario, una mayor independencia, medida según la capacidad para usar transporte público, se asoció con una menor prevalencia. Los medicamentos para los sistemas cardiovascular, gastrointestinal y endocrino fueron los más utilizados (43).

Por otra parte, se ha visto que los ancianos polimedicados son aquellos dependientes en sus actividades de la vida diaria. Las causas de la dependencia de una persona son múltiples y varían bastante de un individuo a otro, pero muchas propician una polimedicación en los ancianos. La capacidad para auto valerse por sí mismo es considerada de gran importancia a la hora de evaluar el estado de salud de un paciente adulto mayor, y es que esta capacidad va a resumir su calidad de vida (42).

El sedentarismo influye en el desarrollo de los trastornos neurocognitivos, tal y como se menciona en un estudio en el Perú en el 2019 que demostró que con el envejecimiento el cerebro experimenta cambios morfológicamente normales, pero a su vez demostró que el aspecto nutricional tiene un papel fundamental en el buen funcionamiento cerebral. Se encontró que el estado de mal nutrición está asociado al deterioro cognitivo (30).

La hiperglucemia también causa alteraciones cognitivas, como el enlentecimiento en el procesamiento de la información o la disminución de la atención. El grado de control glucémico se ha mostrado relacionado con alteraciones cognitivas en la diabetes mellitus ${ }^{35}$. En el estudio "The Diabetes Control and Complications Trial-DCCT" los pacientes con hemoglobina glicosilada menor de 7,4\% tenían mejor respuesta motora y psicomotora que aquellos con hemoglobina glicosilada mayor a $8,8 \%$ (36). En pacientes con diabetes mellitus hay trabajos que muestran un riesgo 4 veces superior de presentar deterioro cognitivo si los valores de hemoglobina glicosilada son mayores del 7\%. Un estudio muestra que en pacientes con diabetes mellitus y edad avanzada por cada incremento del 1\% de hemoglobina glicosilada disminuye 0,33 puntos la puntuación Minimental State Examination Score. En el presente estudio se encontró que los pacientes con mal control glicémico muestran mayor desarrollo de trastornos neurocognitivos (tabla 3).

Se tuvieron varias limitaciones durante el desarrollo del presente debajo de investigación: La primera limitación seria que el estudio se realizó en pacientes de un hospital lo cual haría que la frecuencia de diabetes mellitus, de trastornos neurocognitivos y otras enfermedades sea mayor; probablemente los resultados tengan cifras mayores con respecto a enfermedades que la población en general. La segunda limitación sería que los participantes del estudio son marinos retirados y sus familias, por lo cual los resultados probablemente no se puedan extrapolar a la población latinoamericana en general. La tercera limitación supone que no se contaban con datos prospectivos de MoCA con lo cual no se ha podido establecer estudios de riesgo. Sin embargo, tenemos un tamaño muestral adecuado que nos permite el desarrollo del estudio.

\section{CONCLUSIONES}

Se encontró una relación entre la diabetes mellitus y los trastornos neurocognitivos.

Ser del sexo masculino constituye un factor protector contra los trastornos neurocognitivos en adultos mayores.

La polifarmacia actúa como predictor para desarrollo de trastorno neurocognitivo en pacientes adultos mayores, los factores que se asocian más a la polifarmacia son las enfermedades crónicas, el grado de dependencia para las actividades básicas de la vida diaria.

Nuestros resultados indicarían una posible asociación entre hiperglucemia y los trastornos neurocognitivos, debiendo resaltar el rol de la hemoglobina glicosilada como herramienta de apoyo para el control de diabetes y su posible asociación con riesgo de TNC en adultos mayores.

\section{Correspondencia}

Daniela Zapata-Tragodara

Correo electrónico: daniela.zapata.tragodara@gmail. com

Declaración de Financiamiento y de Conflictos de Intereses: Este proyecto es autofinanciado, los autores declaramos no tener conflictos de interés.

\section{REFERENCIAS BIBLIOGRAFICAS}

1. Formiga F, Pérez-Maraver M. Diabetes mellitus tipo 3. ¿El renacer de la insulina inhalada? Endocrinol Nutr. 2014; 61 (4): 173-5. doi: 10.1016/ 
Relación entre trastornos neurocognitivos, Diabetes Mellitus Tipo 2 y otros factores en adultos mayores.

j.endonu.2014.03.001

2. Muñoz G, Degen C, Schroder J, Toro P. Diabetes mellitus y su asociación con deterioro cognitivo y demencia. Revista Médica Clínica Las Condes. 2016; 27(2): 266-270. DOI:10.1016/j.rmclc.2016.04.015

3. Gelber R, Launer L, White L. The Honolulu-Asia Aging Study: Epidemiologic and Neuropathologic Research on Cognitive Impairment. Curr Alzheimer Res. 2012; 9(6): 664-672.

4. Custodio N, Montesinos R, Alarcón J. Evolución histórica del concepto y criterios actuales para el diagnóstico de demencia. Rev Neuropsiquiatr . 2018; 81(4): 235-249.

5. Roglic G. WHO Global report on diabetes: A summary. Int J Non-Commun Dis. 2016; 1(1): 3-8.

6. Asociación Latinoamericana de diabetes. Guías ALAD sobre el Diagnóstico, Control y Tratamiento de la Diabetes Mellitus Tipo 2 con Medicina Basada en Evidencia. 2019. Ciudad de Mexico: Revista de la ALAD; 2019.

7. Rueda A, Enríquez L. Una revisión de técnicas básicas de neuroimagen para el diagnóstico de enfermedades neurodegenerativas. Biosalud. 2018; 17(2):59-90. DOI: https://dx.doi.org/10.17151/biosa.2018.17.2.5

8. Zhou T, Hu Z, Yang S, Sun L, Yu Z, Wang, G. Role of adaptive and innate immunity in Type 2 Diabetes Mellitus. Journal of Diabetes Research. 2018; 2018: 1-9. doi:10.1155/2018/7457269

9. Cunha BA. Antibiotic selection for diabetic foot infections: A review. J Foot Ankle Surg. 2000; 39(4): 253-257. doi:10.1016/s1067-2516(00)80009-5

10. Bader MS, Yi Y, Abouchehade K, Haroon B, Bishop LD, Hawboldt J. Community-acquired pneumonia in patients with diabetes mellitus: Predictors of complications and length of hospital stay. Am J Med Sci. 2016; 352(1): 30-35. doi:10.1016/j. amjms.2016.02.032

11. Instituto Nacional de Estadística e Informática. Situación de la población adulta mayor informe técnico $\mathrm{N}^{\mathrm{o}}$ 3. Lima: Instituto Nacional de Estadística e Informática; 2019.

12. Hu C, Yu D, Sun X, Zhang M, Wang L, Qin H. The prevalence and progression of mild cognitive impairment among clinic and community populations: a systematic review and meta-analysis. International Psychogeriatrics. 2017; 29(10): 1595-1608. doi:10.1017/s1041610217000473

13. Ward A, Arrighi HM, Michels S, Cedarbaum JM. Mild cognitive impairment: Disparity of incidence and prevalence estimates. Alzheimer's \& Dementia. 2012; 8(1): 14-21. doi:10.1016/j.jalz.2011.01.002

14. Sosa AL. Prevalence, distribution, and impact of mild cognitive impairment in Latin America, China, and India: A 10/66 population-based study. PLoS Medicine. 2012; 9(2): e1001170. doi:10.1371/ journal.pmed.1001170
15. Luna-Solis Y, Vargas H. Factores asociados con el deterioro cognoscitivo y funcional sospechoso de demencia en el adulto mayor en Lima Metropolitana y Callao. Revista de Neuro-Psiquiatría. 2018; 81(1): 9-19. doi: https://doi.org/10.20453/rnp.v81i1.3269

16. Biessels GJ, Despa F. Cognitive decline and dementia in diabetes mellitus: mechanisms and clinical implications. Nat Rev Endocrinol. 2018;14(10):591604. doi: 10.1038/s41574-018-0048-7

16. Zilliox LA, Chadrasekaran K, Kwan JY, Russell JW. (2016). Diabetes and Cognitive Impairment. Curr Diab Rep. 2016;16(9):87. doi: 10.1007/s11892-0160775-x.

17. Kodl CT, Seaquist ER. Cognitive dysfunction and Diabetes Mellitus. Endocrine Reviews. 2008; 29(4): 494-511. doi:10.1210/er.2007-0034

18. Lalithambika CV, Arun CS, Saraswathy LA, Bhaskaran R. Cognitive impairment and its association with glycemic control in type 2 diabetes mellitus patients. Indian J Endocrinol Metab. 2019; 23(3): 353-356. doi: 10.4103/ijem.IJEM 2419

19. Jacob L, Bohlken J, Kostev K. Risk Factors for mild cognitive impairment in German primary care practices. Journal of Alzheimer's Disease. 2017; 56(1): 379-384. doi:10.3233/jad-160875

20. Andersen SL, Sweigart B, Sebastiani P, Drury J, Sidlowski S, Perls TT. Reduced prevalence and incidence of cognitive impairment among centenarian offspring. J Gerontol A Biol Sci Med Sci. 2019;74(1):108-113. doi: 10.1093/gerona/gly141

21. Zhang X, Jiang X, Han S, Liu Q, Zhou J. Type 2 Diabetes Mellitus Is Associated with the Risk of Cognitive Impairment: a Meta-Analysis. J Mol Neurosci. 2019; 68(2):251-260. doi: 10.1007/s12031019-01290-3

22. Roberts RO, Geda YE, Knopman DS, et al. Association of duration and severity of diabetes mellitus with mild cognitive impairment. Arch Neurol. 2008; 65(8): 1066-73. doi: 10.1001/ archneur.65.8.1066

23. Saito S, Ohi T, Murakami T, et al. Association between tooth loss and cognitive impairment in communitydwelling older Japanese adults: a 4-year prospective cohort study from the Ohasama study. BMC Oral Health. 2018;18(1): 142. doi: 10.1186/s12903-0180602-7.

24. Winkler A, Dlugaj M, Weimar C, et al. Association of diabetes mellitus and mild cognitive impairment in middle-aged men and women. Journal of Alzheimer's Disease. 2014; 42(4): 1269-1277. doi:10.3233/jad-140696

25. Saedi E, Gheini MR, Faiz F, Arami MA. Diabetes mellitus and cognitive impairments. World Journal of Diabetes. 2016; 7(17): 412. doi:10.4239/wjd. v7.i17.412

26. Guadalupe M, Rueda M. Asociación entre puntajes 
bajosn test neuropsicológicos y niveles de vitamina $\mathrm{D}$, determinantes clínico-geriátricas en pacientes adultos mayores ambulatorios que acuden a las jornadas de atención del Hospital de Atención Integral del Adulto Mayor, en el segundo trimestre del año 2018. Tesis de Especialidad. Quito: Pontifica Universidad Catolica de Ecuador; 2019.

27. Runzer-Colmenares FM, Samper-Ternent R, Al Snih S, Ottenbacher KJ, Parodi JF, Wong R. Prevalence and factors associated with frailty among Peruvian older adults. Archives of gerontology and geriatrics. 2014; 58(1): 69-73. https://doi. org/10.1016/j.archger.2013.07.005

28. Formiga F, Rodríguez L. Diabetes, depresión y demencia. Tres «d» interrelacionadas en las personas mayores. Rev Esp Geriatr Gerontol. 2015 ;50(3):1078.

29. Formiga F, Rodríguez L. Paciente anciano con diabetes mellitus y fragilidad. ¿Asociación siempre presente? Rev Esp Geriatr Gerontol. 2014 ;49(6):2534.

30. Vega-Quintana A, Moreno-Cervantes CA, PradoHernández OA, Luna-Torres S, Torres-Gutiérrez JL. Comorbilidad en pacientes mayores de 65 años con demencia. Med Int Méx. 2018;34(6):848-854. DOI: https://doi.org/10.24245/mim. v34i6.2123

31. Bernabeu-Wittle M, Diez J, Nieto-Martín D, RamirezDuque N, Ollero M. Simplification of the Barthel scale for screening for fraility and severe dependency in polypathological patients. Revista Clinica Espanola. 2019, 219(8):433-439.

32. Saenz-Miguel S, Runzer-Colmenares F, Parodi J. Trastornos depresivos en adultos mayores de once comunidades altoandinas del Perú, 2013-2017. Acta Médica Peruana. 2019; 36(1): 26-31.

33. Gutiérrez-Valencia M, Aldaz Herce P, LacalleFabo E, Contreras B, Cedeno-Veloz B, MartínezVelilla N. Prevalencia de polifarmacia y factores asociados en adultos mayores en España: Datos de la Encuesta Nacional de Salud 2017. Med clín. 2019; 153(4): 141-150.

34. Rothman M, Leo-Summers L, Gill T. Prognostic significance of potential frailty criteria. J Am Geriatr Soc. 2008; 56(12): 2211-2116. doi:10.1111/j.15325415.2008.02008. $\mathrm{x}$
35. Patnode CD, Perdue LA, Rossom RC, et al. Screening for cognitive impairment in older adults: Updated evidence report and systematic review for the US preventive services task force. JAMA. 2020;323(8):764-785. doi: 10.1001/jama.2019.22258

36. Gao L, Matthews FE, Sargeant LA, Brayne C. Cognitive Function and Ageing Study. An investigation of the population impact of variation in HbA1c levels in older people in England and Wales: from a population-based multi-centre longitudinal study. BMC Public Health. 2008; 8:54-65.

37. Mrak P, Rakovac I, Moarak A, Kienreich K, Fritz C, Pieber TR, et al. Correlates of cognitive function measured in frail elderly patients with diabetes mellitus. Diabetologia. 2009;52 Suppl 1:S1-55

38. Jurado M, Santibáñez R, Palacios-Mendoza M, et al. Deterioro Cognitivo en pacientes diabéticos entre 55 a 65 años de edad. Reporte final de estudio observacional, transversal en la ciudad de Guayaquil. Revista Ecuatoriana de Neurología. 2018; 27(1): 4150.

39. Castro-Rodríguez J, Orozco-Hernández J, MarínMedina D. Polifarmacia y prescripción de medicamentos potencialmente no apropiados en ancianos. Rev Méd Risaralda. 2016; 22 (1): 52-57.

40. Cano-Guitierrez C, Samper-Ternent R, Cabrera J, Rosselli D. Uso de medicamentos en adultos mayores de Bogotá, Colombia. Revista Peruana de Medicina Experimental y Salud Publica. 2016; 33(3): 419-424. Doi: https://dx.doi.org/10.17843/rpmesp.2016.333. 2292

41. Martínez C, Pérez V, Carballo M, Larrondo J. Polifarmacia en los adultos mayores. Revista Cubana de Medicina General Integral. 2005; 21:1-2.

42. Serra M, Germán J. Caracterización de adultos mayores con polifarmacia evaluados en la consulta de geriatría. Revista Cubana de Medicina Militar. 2014; 43(3): 285-292.

43. Bazalar-Silva L, Runzer-Colmenares F, Parodi J. Asociación entre el estado nutricional según índice de masa corporal y deterioro cognitivo en adultos mayores del Centro Médico Naval del Perú, 20102015. Acta méd Peru. 2019; 36(1): 5-10. DOI: https:// doi.org/10.35663/amp.2019.361.628

Recibido: 15/04/2020

Aceptado: 16/06/2020 